\title{
On the Existence and Uniqueness of Solution to Fractional-Order Langevin Equation
}

\author{
Ahmed Salem $\mathbb{D}^{1}$ and Noorah Mshary ${ }^{1,2}$ \\ ${ }^{1}$ Department of Mathematics, Faculty of Science, King Abdulaziz University, P.O. Box 80203, Jeddah 21589, Saudi Arabia \\ ${ }^{2}$ Department of Mathematics, Faculty of Science, Jazan University, P.O. Box 45142, Jazan 2097, Saudi Arabia \\ Correspondence should be addressed to Ahmed Salem; ahmedsalem74@hotmail.com
}

Received 29 August 2020; Revised 27 September 2020; Accepted 15 October 2020; Published 30 October 2020

Academic Editor: Soheil Salahshour

Copyright (c) 2020 Ahmed Salem and Noorah Mshary. This is an open access article distributed under the Creative Commons Attribution License, which permits unrestricted use, distribution, and reproduction in any medium, provided the original work is properly cited.

In this work, we give sufficient conditions to investigate the existence and uniqueness of solution to fractional-order Langevin equation involving two distinct fractional orders with unprecedented conditions (three-point boundary conditions including two nonlocal integrals). The problem is introduced to keep track of the progress made on exploring the existence and uniqueness of solution to the fractional-order Langevin equation. As a result of employing the so-called Krasnoselskii and Leray-Schauder alternative fixed point theorems and Banach contraction mapping principle, some novel results are presented in regarding to our main concern. These results are illustrated through providing three examples for completeness.

\section{Introduction}

Unquestionably, the fractional calculus, which requires the order of the functional operator of calculus to be turned into the fractional case, is a forceful mathematical argument for providing much and more flexibility in dealing with many real-world applications [1, 2]. More particularly, the fractional-order differential equations (FODEs), which are considered a cornerstone of this field, have been extensively employed in modeling several phenomena and numerous scientific applications such as diffusion modeling [3], robot manipulators [4], economics [5], and many more [6-9]. In view of its outstanding importance, lots of mathematicians have paid their attentions to deeply explore the initial and/or boundary value problems (BVPs) established based on such equations (see [10-13]). Actually, these problems, which may have different boundary conditions, describe several physical real-world issues like thermoelasticity, underground water flow, and heat conduction. One of the most significant of those problems is known as the Langevin equation which was formulated for the first time in 1908 by Langevin, who still much earlier described the progress of some physical matters in fluctuating settings $[14,16]$. It came out, later on, that such equation had assisted physicians successfully to identify some techniques related to the abnormal diffusion. It also, afterward, succeeded in describing some other techniques in economic field that contain the cost index operating [17]. Besides, its generalization played, and still plays, a significant duty in describing the noise causes in the field of critical dynamics [18]. In 1996, Mainardi and Pironi fractionalized such equation and hence turned it into the fractional-order case to be named later the fractional-order Langevin equation (FoLE) [19]. Several articles then made this equation up at the top of their works (see, e.g., [2022]). It has been recently shown that such equation, for example, but not limited to, plays notable role in outlining the reaction-diffusion models $[23,24]$ and describes some processes associated with porous media [25, 26].

In the same framework, many efforts have been underway to explore the two most main aspects associated with the solutions of the FoLEs: the existence and uniqueness [27]. In view of studying such two aspects, several recent articles have been published. In particular, through using some special boundary conditions, some applicable results related to the existence of solutions for the FoLE have been reported in [28]. Both aspects have been investigated when the 
coupled incommensurate fractional-order systems were handled by Salem and Alnegga and Salem et al. in [15, 29]. However, in more recent times, numerous outcomes associated with those aspects have, moreover, been informed widely (see $[1,14,21,30-34])$. In more concrete terms, some useful theoretical tools, like the so-called Banach contraction principle and Krasnoselskii's fixed point theorem, have been employed to explore the existence and uniqueness of solutions for several nonlinear FODEs such as the implicit FODEs [35], the fractional-order Cauchy problems [36].

As per the best of our knowledge, there are few published works that address the FoLE with nonlocal integral boundary conditions as informed in [14]. In view of this motivation, this work examines the existence and uniqueness of solution for the nonlinear FoLE with three-point boundary conditions involving two nonlocal integrals. Actually, this boundary value problem, with two different values of fractional order, has the following form:

$$
{ }^{c} D^{r}\left({ }^{c} D^{s}+\ell\right) z(t)=f\left(t, z(t),{ }^{c} D^{\rho} z(t)\right), t \in[0, T],
$$

where ${ }^{c} D^{r}$ is the Caputo derivative operator of order $r$ in which $k-1<r \leq k$ and $k \in \mathbb{N}$. In this work, we assume that the function $f:[0, T] \times \mathbb{R} \times \mathbb{R} \rightarrow \mathbb{R}$ is a continuous function, and $1<r<2,0<\rho \leq s \leq 1$, and $\ell \in \mathbb{R}$ are the dissipative parameters. We take here the function $f$, in general form, constitutes from position (displacement) $z(t)$ and fractional velocity ${ }^{c} D^{\rho} z(t)$ of the particle at time $t \in[0, T], T>0$. This function perhaps contains external force field, positiondependent phenomenological fluid friction coefficient, intensity of the stochastic force, or zero-mean Gaussian white noise term.

The equation (1) subjects to the following boundary conditions:

$$
z(0)=0, z(T)=\alpha \int_{0}^{T} z(u) d u, z(\zeta)=\beta \int_{0}^{\zeta} z(u) d u,
$$

where $\alpha, \beta \in \mathbb{R}$ and $0<\zeta<T$. It is remarkable to see that the first condition indicates the particle begins its motion from the origin point. Also, all conditions can be interpreted informally as "the displacement of the particle at a certain point proportionates the signed area of the region in the $t z$-plane that is bounded by the graph of $z$ and the $t$-axis from the origin to this certain point".

One more observation should be recorded; all hypotheses and conditions in this work are assumed on the basis of the various views inspired by the aforementioned cited articles. Anyhow, here is how the rest of this paper is arranged. Section 2 introduces some primary preliminaries associated with fractional calculus and some fixed point theorems, while Section 3 deduces some auxiliary lemmas that pave the way for establishing novel theorems related to the existence and uniqueness of the solution for our main problem. Such theorems are illustrated and shown in Section 4, while Section 5 involves one illustrative example to verify all findings, followed by the last section that summarizes the achievements of the whole work.

\section{Preliminaries}

In the whole of this work, the Caputo derivative operator and its inverse operator, the Riemann-Liouville (R-L) integral, are adopted since they suite most physical systems. These two operators as well as some basic concepts and essential preliminaries about fractional calculus are briefly presented here together with some theoretical results associated with the fixed point theorem.

Definition 1. [37]. The R-L integral operator of order $r$ for a continuous function $z:[0, \infty) \rightarrow \mathbb{R}$ is outlined as

$$
I^{r} z(t)=\frac{1}{\Gamma(r)} \int_{0}^{t}(t-v)^{r-1} z(v) d v
$$

where $r>0$.

Definition 2. [37]. The R-L derivative operator of order $r$ for a $k$-times absolutely continuous function $z:[0, \infty) \rightarrow \mathbb{R}$ is outlined as

$D^{r} z(t)=\frac{1}{\Gamma(k-r)}\left(\frac{d}{d t}\right)^{k} \int_{0}^{t}(t-v)^{k-r-1} z(v) d v=\left(\frac{d}{d t}\right)^{k} I^{k-r} z(t)$,

where $k-1 \leq r<k$ and $k \in \mathbb{N}$.

Definition 3. [37]. The Caputo derivative operator of order $r$ for a $k$-times absolutely continuous function $z:[0, \infty) \rightarrow \mathbb{R}$ is outlined as

$$
{ }^{c} D^{r} z(t)=\frac{1}{\Gamma(k-r)} \int_{0}^{t}(t-v)^{k-r-1} z^{(k)}(v) d v
$$

where $k-1<r \leq k$ and $k \in \mathbb{N}$.

The following remark states some important relations chosen from the famous books [37]. Actually, such relations will be used during the whole of this work.

Remark 4. Suppose that $z:[0, \infty) \rightarrow \mathbb{R}$ is a continuous function, $r$ is a positive real. Then,

(i) $I^{r} I^{s} z(t)=I^{r+s} z(t), s>0$

$(t)$

(ii) ${ }^{c} D^{r} I^{s} z(t)=I^{s-r} z(t)$, for $r \in[0, s]$, while ${ }^{c} D^{r} I^{r} z(t)=z$

(iii) $I^{r} t^{\rho}=\Gamma(\rho+1) \Gamma(\rho+r+1) t^{\rho+r}, \rho>-1$ $[r]$

(iv) ${ }^{c} D^{r} t^{\rho}=\Gamma(\rho+1) \Gamma(\rho-r+1) t^{\rho-r}, \rho>-1, \rho \neq 0,1, \cdots$,

(v) ${ }^{c} D^{r} t^{\rho}=0, \rho=0,1, \cdots,[r]$

Lemma 5. Suppose ${ }^{c} D^{r} z$ is a continuous function, then

$$
I^{r c} D^{r} z(t)=z(t)+c_{0}+c_{1} t+\cdots+c_{k-1} t^{k-1}
$$

for $k \in \mathbb{N}$, where $k-1<r \leq k$. 
Next, some primary keys and essential theorems related to the fixed point theorem are stated for the purpose of getting novel results.

Definition 6. [38, 39]. A contraction of a normed space $\mathbb{X}$ is a mapping $S: \mathbb{X} \rightarrow \mathbb{X}$ that meets

$$
\left\|S z_{1}-S z_{2}\right\| \leq \delta\left\|z_{1}-z_{2}\right\|, \forall z_{1}, z_{2} \in \mathbb{X}
$$

for some $\delta<1$.

Theorem 7. (Banach fixed point theorem, Granas and Dugundji $[38,39])$. There is a unique fixed point for every contraction mapping on a complete metric space.

Theorem 8. (Krasnoselskii fixed point theorem [38]). For a convex set $\mathscr{B}_{\varepsilon}$ of a Banach space $\mathbb{X}$ and a mapping $S z=S_{1} z$ $+\mathrm{S}_{2} z$ such that

(1) $S_{1} z_{1}+S_{2} z_{2} \in \mathscr{B}_{\varepsilon}$, for each $z_{1}, z_{2} \in \mathscr{B}_{\varepsilon}$

(2) $S_{2}$ represents a contraction mapping

(3) $S_{1}$ is continuous and compact

Then, $S$ has a fixed point.

Theorem 9. [39]. Let $\mathcal{O}$ be an open set of the Banach space $\mathbb{X}=C\left([0, T], \mathbb{R}^{+}\right)$such that $\mathcal{O} \neq \phi$. If the operator $S: \overline{\mathcal{O}} \rightarrow \mathbb{X}$ is relatively compact, then either

(1) S has a fixed point $z^{*} \in \overline{\mathcal{O}}$, or

(2) There exists $\gamma \in(0,1)$ and $z^{*} \in \partial \mathcal{O}$ such that $\gamma S(z)=z$, where $\partial \mathcal{O}$ is the boundary of $\mathcal{O}$ itself

\section{Some Deduced Auxiliary Lemmas}

In this part, some auxiliary lemmas are introduced in order to pave the way for exploring the existence and uniqueness of the solution for our main problem. In other words, such lemmas will play a major role in transforming the main issue to a fixed point problem. For this purpose, let us introduce the following two results, with noting that the function $f(t$, $\left.z(t),{ }^{c} D^{\rho} z(t)\right)$ given in (1) will be indicated by $f(t)$ for simplicity.

Lemma 10. Let $f \in C([0, T], \mathbb{R})$. Then, the unique root of the problem given in (1)-(2) can be expressed as

$$
z(t)=I^{s+r} f(t)-\ell I^{s} z(t)+\chi_{1}(t) \Lambda_{\alpha}(T)+\chi_{2}(t) \Lambda_{\beta}(\zeta),
$$

where

$$
\chi_{1}(t)=\frac{t^{s}(t-\zeta)}{(T-\zeta) T^{s}}
$$

$$
\begin{gathered}
\chi_{2}(t)=\frac{t^{s}(T-t)}{(T-\zeta) \zeta^{s}}, \\
\Lambda_{\alpha}(t)=\alpha I z(t)-I^{s+r} f(t)+\ell I^{s} z(t) .
\end{gathered}
$$

Proof. Applying the integral operator $I^{r}$ to both sides of (1) and then using Lemma 5. yield

$$
{ }^{c} D^{s} z(t)=-\ell I z(t)+I^{r} f(t)+c_{2} t+c_{1} .
$$

Again, applying $I^{r}$ to both sides of (12) gives

$$
z(t)=I^{s+r} f(t)-\ell I^{s} z(t)+\frac{c_{2} t^{s+1}}{\Gamma(s+2)}+\frac{c_{1} t^{s}}{\Gamma(s+1)}+c_{0},
$$

where $c_{i}$ 's are arbitrary constants, and $i=1,2,3$. Applying the first condition of (2) yields $c_{0}=0$, and hence

$$
z(t)=I^{s+r} f(t)-\ell I^{s} z(t)+\frac{c_{2} t^{s+1}}{\Gamma(s+2)}+\frac{c_{1} t^{s}}{\Gamma(s+1)} .
$$

By using the two other conditions given in (2), we obtain the following:

$$
\begin{aligned}
& \frac{c_{1} T^{s}}{\Gamma(s+1)}+\frac{c_{2} T^{s+1}}{\Gamma(s+2)}=\Lambda_{\alpha}(T) \\
& \frac{c_{1} \zeta^{s}}{\Gamma(s+1)}+\frac{c_{2} \zeta^{s+1}}{\Gamma(s+2)}=\Lambda_{\beta}(\zeta) .
\end{aligned}
$$
ing:

Solving the two equations (15) and (16) gives the follow-

$$
\begin{gathered}
\frac{c_{1}}{\Gamma(s+1)}=\frac{T}{(T-\zeta) \zeta^{s}} \Lambda_{\beta}(\zeta)-\frac{\zeta}{(T-\zeta) T^{s}} \Lambda_{\alpha}(T), \\
\frac{c_{2}}{\Gamma(s+2)}=\frac{1}{(T-\zeta) T^{s}} \Lambda_{\alpha}(T)-\frac{1}{(T-\zeta) \zeta^{s}} \Lambda_{\beta}(\zeta) .
\end{gathered}
$$

Substituting (17) and (18) into (14) yields the result. Conversely, it is easy by aiding with Remark 4 . and Lemma 5 . to see that the solution (8) satisfies the fractional differential equation (1) and the boundary conditions in (2).

Lemma 11. Let $\chi_{1}$ and $\chi_{2}$ be defined as given in (9) and (10), respectively. Then,

$$
\begin{gathered}
\chi_{1}=\max _{t \in[0, T]}\left|\chi_{1}(t)\right|=\max \left\{\frac{\zeta^{s+1} s^{s}}{(s+1)^{s+1}(T-\zeta) T^{s}}, 1\right\}, \\
\chi_{2}=\max _{t \in[0, T]}\left|\chi_{2}(t)\right|=\frac{T^{s+1} s^{s}}{(s+1)^{s+1}(T-\zeta) \zeta^{s}} .
\end{gathered}
$$

Proof. Let $t, \zeta \in[0, T]$. Define the function $u(t)$ as follows:

$$
u(t)=t^{s}|t-\zeta|=\left\{t^{s}(\zeta-t), 0 \leq t \leq \zeta, t^{s}(t-\zeta), \zeta \leq t \leq T .\right.
$$


One observes that when $0 \leq t \leq \zeta$, we have $u^{\prime}(t)=t^{s-1}(s$ $\zeta-(s+1) t)$, which means that $u(t)$ is increasing on $\left(0, t_{0}\right)$ and it is decreasing on $\left(t_{0}, \zeta\right)$, where $t_{0}=s \zeta /(s+1)$. Hence, we can deduce that $u(t)$ has a maximum value at $t=t_{0}$. On the other hand, when $\zeta<t \leq T$, we obtain $u^{\prime}(t)=t^{s-1}((s+1$ $t-s \zeta)$, which implies that $u(t)$ is increasing on $(\zeta, T)$, and so $u(t)$ has a maximum value at $t=T$. Therefore, the result holds due to the following:

$$
\chi_{1}=\max \left\{\frac{u\left(t_{0}\right)}{(T-\zeta) T^{s}}, \frac{u(T)}{(T-\zeta) T^{s}}\right\}
$$

In similar manner, one can deduce the second statement of this lemma.

To follow up on the progress made in exploring the existence and uniqueness of solutions for the FoLEs, some further results are established below, which will surely serve us in the next sections. In this regard, let $\mathscr{C}=C([0, T], \mathbb{R})$ be Banach space of continuous real-valued functions equipped with the usual supremum norm

$$
\|z\|=\sup _{0 \leq t \leq T}|z(t)|, z \in \mathscr{C}
$$

Define the following set

$$
\mathscr{P}=\left\{z(t): z(t) \in \mathscr{C},{ }^{c} D^{\rho} z(t) \in \mathscr{C}\right\}, t \in[0, T] .
$$

One can easily verify that $\mathscr{P}$ is a Banach subspace equipped with the following norm:

$$
\begin{aligned}
\|z\|_{\mathscr{P}} & =\max \left\{\|z\|,\left\|{ }^{c} D^{\rho} z\right\|\right\} \\
& =\max \left\{\sup _{0 \leq t \leq T}|z(t)|, \sup _{0 \leq t \leq T} \mid D^{c} D^{\rho}(z(t) \mid\} .\right.
\end{aligned}
$$

In view of Lemma 10, the main problem given in (1)-(2) will be transformed into its equivalent fixed point problem. In other words, the function $z(t)$ should satisfy the two statements; $S z(t)=z(t)$ and ${ }^{c} D^{\rho} S z(t)={ }^{c} D^{\rho} z(t)$, for all $t \in[0, T]$, where $S: \mathscr{C} \rightarrow \mathscr{C}$ is an operator defined on the Banach space $\mathscr{C}$. In order to achieve this goal, consider the operator $S$ as defined a little while ago. That is,

$$
\begin{gathered}
(S z)(t)=I^{s+r} f(t)-\ell I^{s} z(t)+\chi_{1}(t) \Lambda_{\alpha}(T)+\chi_{2}(t) \Lambda_{\beta}(\zeta) \\
\left({ }^{c} D^{\rho} S z\right)(t)= \\
\quad I^{s+r-\rho} f(t)-\ell I^{s-\rho} z(t) \\
+\chi_{3}(t) \Lambda_{\alpha}(T)+\chi_{4}(t) \Lambda_{\beta}(\zeta)
\end{gathered}
$$

where $\Lambda_{\alpha}(\cdot)$ is defined in (11) and

$$
\chi_{3}(t)={ }^{c} D^{\rho} \chi_{1}(t)=\frac{\Gamma(s+2) t^{s-\rho}}{\Gamma(s-\rho+2)(T-\zeta) T^{s}}\left[t-\frac{\zeta(s+1-\rho)}{s+1}\right],
$$

$\chi_{4}(t)={ }^{c} D^{\rho} \chi_{2}(t)=-\frac{\Gamma(s+2) t^{s-\rho}}{\Gamma(s-\rho+2)(T-\zeta) \zeta^{s}}\left[t-\frac{T(s+1-\rho)}{s+1}\right]$

Next, we introduce the following auxiliary result based on all provided aforementioned principles.

Lemma 12. Let $\chi_{3}(t)$ and $\chi_{4}(t)$ are defined as in (28) and (29), respectively. Then,

$$
\begin{aligned}
\chi_{3}= & \left\|\chi_{3}\right\| \\
= & \frac{\Gamma(s+1)}{\Gamma(s-\rho+2)(T-\zeta)} \max \\
& \cdot\left\{\frac{\zeta^{s-\rho+1}}{T^{s}}\left(\frac{s-\rho}{s+1}\right)^{s-\rho}, \frac{(s+1) T-(s-\rho+1) \zeta}{T^{\rho}}\right\}, \\
\chi_{4}= & \left\|\chi_{4}\right\|=\frac{\Gamma(s+1) T^{s-\rho+1}}{\Gamma(s-\rho+2)(T-\zeta) \zeta^{s}} \max \left\{\left(\frac{s-\rho}{s+1}\right)^{s-\rho}, \rho\right\} .
\end{aligned}
$$

Proof. Let $t_{0}=\zeta(s+1-\rho) /(s+1)$. Define the function

$$
u(t)=t^{s-\rho}\left|t-t_{0}\right|, t \in[0, T] .
$$

If $0 \leq t \leq t_{0}$, then $u(t)=t^{s-\rho}\left(t_{0}-t\right)$ and

$$
u^{\prime}(t)=t^{s-\rho-1}\left[(s-\rho) t_{0}-(s-\rho+1) t\right]
$$

which leads to $u(t)$ is increasing on $\left(0, t_{1}\right)$ and is decreasing on $\left(t_{1}, t_{0}\right)$ where

$$
t_{1}=s-\rho s-\rho+1 t_{0}=\zeta(s-\rho) s+1<t_{0} .
$$

This concludes that $u\left(t_{1}\right)$ is a maximum value.

If $t_{0}<t \leq T$, then $u(t)=t^{s-\rho}\left(t-t_{0}\right)$ and

$$
u^{\prime}(t)=t^{s-\rho-1}\left[(s-\rho+1) t-(s-\rho) t_{0}\right]>t^{s-\rho-1} t_{0}>0
$$

which leads to $u(t)$ is increasing on $\left(t_{0}, T\right)$. This concludes that $u(T)$ is a maximum value. In similar manner, one can deduce the second statement of this lemma.

\section{Existence and Uniqueness Results}

This section proposes some theoretical results related to the existence and uniqueness of the solution for the main target problem, the FoLE given in (1)-(2). First of all, we establish some mathematical assumptions that will be used from now on. Such assumptions are given below.

$$
\begin{array}{r}
\Delta=|\ell| \Delta(s)+|\alpha| \chi_{1} T+|\beta| \chi_{2} \zeta, \\
\Delta^{\prime}=|\ell| \Delta^{\prime}(s)+|\alpha| \chi_{3} T+|\beta| \chi_{4} \zeta .
\end{array}
$$

where

$$
\Delta(s)=\left(1+\chi_{1}\right) T^{s}+\chi_{2} \zeta^{s} \Gamma(s+1),
$$




$$
\Delta^{\prime}(s)=T^{s-\rho} \Gamma(s-\rho+1)+\chi_{3} T^{s}+\chi_{4} \zeta^{s} \Gamma(s+1),
$$

and $\chi_{i}, i=1,2,3,4$ are given in (19), (20), (30), and (31), respectively.

In the same vein, we also introduce some further conditions below to establish our proposed results. In fact, these conditions have been recently introduced in [40] for the purpose of investigating the same issue of ours, but they were adopted for handling another problem.

$\mathbf{H}_{1}$ The function $f\left(t, z(t),{ }^{c} D^{\rho} z(t)\right)$ is continuous on $[0, T]$ that

$\mathbf{H}_{2}$ There exist a positive function $\psi:[0, T] \rightarrow \mathbb{R}^{+}$such

$$
\left|f\left(t, z(t),{ }^{c} D^{\rho} z(t)\right)\right| q(t)
$$

$\mathbf{H}_{3}$ There exist two constants $M_{1}, M_{2}>0$ such that

$$
\begin{aligned}
& \left|f\left(t, z_{1}(t),{ }^{c} D^{\rho} z_{1}\right)-\mathrm{f}\left(t, z_{2},{ }^{c} D^{\rho} z_{2}\right)\right| \\
& \quad \leq M_{1}\left|z_{1}-z_{2}\right|+M_{2}\left|c_{D^{\rho} z_{1}-{ }^{c} D^{\rho} z_{2}}\right|, t \in[0, T]
\end{aligned}
$$

$\mathbf{H}_{4}$ There exist a positive function $h:[0, T] \rightarrow \mathbb{R}^{+}$and other nondecreasing function $\Psi: \mathbb{R}^{+} \rightarrow \mathbb{R}^{+}$such that

$$
\left|f\left(t, z(t),{ }^{c} D^{\rho} z(t)\right)\right| \leq h(t) \Psi\left(\|z\|_{\mathscr{P}}\right), t \in[0, T]
$$

On the other side, by going back to (26) and (27), one can rewrite each of $S z(t)$ and ${ }^{c} D^{\rho} S z(t)$, as a sum of two operators $S_{1}$ and $S_{2}$. In other words, $S z(t)=\left(S_{1} z+S_{2} z\right)(t)$ and ${ }^{c} D^{\rho} S z($ $t)=\left({ }^{c} D^{\rho} S_{1} z+{ }^{c} D^{\rho} S_{2} z\right)(t)$, where

$$
\begin{aligned}
\left(S_{1} z\right)(t)= & I^{s+r} f(t)-\chi_{1}(t) I^{s+r} f(T)-\chi_{2}(t) I^{s+r} f(\zeta), \\
\left(S_{2} z\right)(t)= & -\ell I^{s} z(t)+\chi_{1}(t)\left[\alpha I z(T)+\ell I^{s} z(T)\right] \\
& +\chi_{2}(t)\left[\beta I z(\zeta)+\ell I^{s} z(\zeta)\right], \\
\left({ }^{c} D^{\rho} S_{1} z\right)(t)= & I^{s+r-\rho} f(t)-\chi_{3}(t) I^{s+r} f(T)-\chi_{4}(t) I^{s+r} f(\zeta), \\
\left({ }^{c} D^{\rho} S_{2} z\right)(t)= & -\ell I^{s-\rho} z(t)+\chi_{3}(t)\left[\alpha I z(T)+\ell I^{s} z(T)\right] \\
& +\chi_{4}(t)\left[\beta I z(\zeta)+\ell I^{s} z(\zeta)\right] .
\end{aligned}
$$

After this preparation, we find ourselves ready to present the results that are at the heart of the matter. Here is the first one that deduced on the basis of the Krasnoselskii theorem.

Theorem 13. Suppose $\mathbf{H}_{1}$ and $\mathbf{H}_{2}$ hold. If

$$
\max \left\{\Delta, \Delta^{\prime}\right\}<1
$$

where $\Delta$ and $\Delta^{\prime}$ are defined in (36) and (37), respectively, then the problem given in (1)-(2) has at least one solution on $[0, T]$.

Proof. To prove this theorem, define the closed ball, $\mathscr{B}_{\varepsilon}$, as follows:

$$
\mathscr{B}_{\varepsilon}=\left\{z(t) \in \mathscr{P}:{ }^{c} D^{\rho} z(t) \in \mathscr{P},\|z(t)\|_{\mathscr{P}} \leq \varepsilon\right\},
$$

where $\mathscr{P}$ and $\|z\|_{\mathscr{P}}$ are defined in Section 3, with radius

$$
\varepsilon \geq\|q\| \max \left\{\Delta(s+r), \Delta^{\prime}(s+r)\right\} 1-\max \left\{\Delta, \Delta^{\prime}\right\},
$$

where $\Delta(\cdot)$ and $\Delta^{\prime}(\cdot)$ are defined in (38) and (39), respectively.

One can easily verify that $\mathscr{B}_{\varepsilon}$ is a convex closed set. However, for the purpose of implementing on the Krasnoselskii theorem given in 2.2, we need firstly to show that $S_{1} z_{1}+S_{2}$ $z_{2} \in \mathscr{B}_{\varepsilon}$, for each $z_{1}(t), z_{2}(t) \in \mathscr{B}_{\varepsilon}$. To carry out this step, we begin with

$$
\begin{aligned}
\left|S_{1} z_{1}\right| \leq & \left|I^{s+r} f\left(t, z_{1}(t), D^{\rho} z_{1}(t)\right)\right| \\
& +\left|\chi_{1}(t)\right|\left|I^{s+r} f\left(T, z_{1}(T), D^{\rho} z_{1}(T)\right)\right| \\
& +\left|\chi_{2}(t)\right|\left|I^{s+r} f\left(\zeta, z_{1}(\zeta), D^{\rho} z_{1}(\zeta)\right)\right| \\
\leq & q(t) \Gamma(s+r)\left[\int_{0}^{t}(t-v)^{s+r-1} d v\right. \\
& \left.+\left|\chi_{1}(t)\right| \int_{0}^{T}(T-v)^{s+r-1} d v+\left|\chi_{2}(t)\right| \int_{0}^{\zeta}(\zeta-v)^{s+r-1} d v\right] \\
= & {\left[t^{s+r}+\left|\chi_{1}(t)\right| T^{s+r}+\left|\chi_{2}(t)\right| \zeta^{s+r}\right] \Gamma(s+r+1)|| q(t) \mid } \\
\leq & \left(1+\left|\chi_{1}(t)\right|\right)\left|T^{s+r}+\right| \chi_{2}(t)\left|\zeta^{s+r} \Gamma(s+r+1)\right| q(t) \mid, \\
\left|S_{2} z_{2}\right| \leq \leq & |\ell| I^{s}\left|z_{2}(t)\right|+\left|\chi_{1}(t)\right|\left[|\alpha| I\left|z_{2}(T)\right|+|\ell| I^{s}\left|z_{2}(T)\right|\right] \\
& +\left|\chi_{2}(t)\right|\left[|\beta| I\left|z_{2}(\zeta)\right|+|\ell| I^{s}\left|z_{2}(\zeta)\right|\right] \\
\leq & {\left[\left(1+\left|\chi_{1}(t)\right|\right) T^{s}+\left|\chi_{2}(t)\right| \zeta^{s} \Gamma(s+1)|\ell|\right.} \\
& \left.+|\alpha|\left|\chi_{1}(t)\right| T+|\beta|\left|\chi_{2}(t)\right| \zeta\right]\left|z_{2}\right| .
\end{aligned}
$$

Due to $z_{2}(t) \in \mathscr{B}_{\mathcal{E}}$, then $\left|z_{2}(t)\right| \leq\left\|z_{2}(t)\right\| \leq\left\|z_{2}(t)\right\|_{\mathscr{P}} \leq \varepsilon$. This matter implies to

$$
\begin{aligned}
\left|S_{1} z_{1}+S_{2} z_{2}\right| \leq & \left(1+\chi_{1}\right) \mid T^{s+r}+\chi_{2} \zeta^{s+r} \Gamma(s+r+1)\|q\|+\left[\left(1+\chi_{1}\right) T^{s}\right. \\
& \left.+\chi_{2} \zeta^{s} \Gamma(s+1)|\ell|+|\alpha| \chi_{1} T+|\beta| \chi_{2} \zeta\right] \varepsilon \\
= & \|q\| \Delta(s+r)+\left(|\ell| \Delta(s)+|\alpha| \chi_{1} T+|\beta| \chi_{2} \zeta\right) \varepsilon \\
= & \|q\| \Delta(s+r)+\Delta \varepsilon .
\end{aligned}
$$

Thus, the last inequality implies $\left\|S_{1} z_{1}+S_{2} z_{2}\right\| \leq \varepsilon$. In a similar manner, we can show that

$$
\begin{aligned}
\left|{ }^{c} D^{\rho} S_{1} z_{1}\right| \leq & \left(T^{s+r-\rho} \Gamma(s+r-\rho+1)+\chi_{3} T^{s+r}\right. \\
& \left.\left.+\chi_{4} \zeta^{s+r}\right] \Gamma(s+r+1)\right)\|q\|, \\
\left|{ }^{c} D^{\rho} S_{2} z_{2}\right| \leq & {\left[\left(T^{s-\rho} \Gamma(s-\rho+1)+\chi_{3} T^{s}\right.\right.} \\
& \left.\left.+\chi_{4} \zeta^{s} \Gamma(s+1)\right)|\ell|+|\alpha| \chi_{3} T+|\beta| \chi_{4} \zeta\right] \varepsilon,
\end{aligned}
$$

which implies that

$$
\begin{aligned}
\left|{ }^{c} D^{\rho} S_{1} z_{1}+{ }^{c} D^{\rho} S_{2} z_{2}\right| & \leq\|q\| \Delta^{\prime}(s+r)+\left(|\ell| \Delta^{\prime}(s)+|\alpha| \chi_{3} T+|\beta| \chi_{4} \zeta\right) \varepsilon \\
& =\|q\| \Delta^{\prime}(s+r)+\Delta^{\prime} \varepsilon .
\end{aligned}
$$


These conclude that

$$
\begin{aligned}
\left\|S_{1} z_{1}+S_{2} z_{2}\right\|_{\mathscr{P}} & =\max \left\{\sup _{t \in[0, T]}\left|S_{1} z_{1}+S_{2} z_{2}\right|,\left.\sup _{t \in[0, T]}\right|^{c} D^{\rho} S_{1} z_{1}+{ }^{c} D^{\rho} S_{2} z_{2} \mid\right\} \\
& =\max \left\{\|q\| \Delta(s+r)+\Delta \varepsilon,\|q\| \Delta^{\prime}(s+r)+\Delta^{\prime} \varepsilon\right\} \\
& =\|q\| \max \left\{\Delta(s+r), \Delta^{\prime}(s+r)\right\}+\max \left\{+\Delta, \Delta^{\prime}\right\} \varepsilon \leq \varepsilon,
\end{aligned}
$$

which means that $S_{1} z_{1}+S_{2} z_{2} \in \mathscr{B}_{\varepsilon}$ for each $z_{1}(t), z_{2}(t) \in \mathscr{B}_{\varepsilon}$. Thus, the first part of Krasnoselskii Theorem 8 has been shown. The next step is to show that the operator $S_{2}$ is contraction. For this purpose, we estimate $\left|S_{2} z_{1}-S_{2} z_{2}\right|$ as follows:

$$
\begin{aligned}
\left|S_{2} z_{1}-S_{2} z_{2}\right| \leq & \ell I^{s}\left|z_{1}(t)-z_{2}(t)\right|+\left|\chi_{1}(t)\right|\left(|\alpha| I\left|z_{1}(T)-z_{2}(T)\right|\right. \\
& \left.+\ell I^{s}\left|z_{1}(T)-z_{2}(T)\right|\right)+\left|\chi_{2}(t)\right|\left(|\beta| I \mid z_{1}(\zeta)\right. \\
& \left.-z_{2}(\zeta)\left|+\ell I^{s}\right| z_{1}(\zeta)-z_{2}(\zeta) \mid\right) \\
\leq & {\left[\left(1+\chi_{1}\right) T^{s}+\chi_{2} \zeta^{s} \Gamma(s+1)|\ell|\right.} \\
& \left.+|\alpha| \chi_{1} T+|\beta| \chi_{2} \zeta\right]\left|z_{1}-z_{2}\right| \\
\leq & {\left[|\ell| \Delta(s)+|\alpha| \chi_{1} T+|\beta| \chi_{2} \zeta\right]|| z_{1}-z_{2} \|_{\mathscr{P}} } \\
= & \Delta\left\|z_{1}-z_{2}\right\|_{\mathscr{P}} .
\end{aligned}
$$

Similarly, we can prove that

$$
\begin{aligned}
\left|{ }^{c} D^{\rho} S_{2} z_{1}-{ }^{c} D^{\rho} S_{2} z_{2}\right| & \\
\leq & {\left[|\ell| \Delta^{\prime}(s)+|\alpha| \chi_{3} T+|\beta| \chi_{4} \zeta\right] \| z_{1} } \\
& -z_{2}\left\|_{\mathscr{P}}=\Delta^{\prime}\right\| z_{1}-z_{2} \|_{\mathscr{P}} .
\end{aligned}
$$

These lead to

$$
\left\|S_{2} z_{1}-S_{2} z_{2}\right\|_{\mathscr{P}} \leq \max \left\{\Delta, \Delta^{\prime}\right\}\left\|z_{1}-z_{2}\right\|_{\mathscr{P}}
$$

which implies that $S_{2}$ is contraction if $\max \left\{\Delta, \Delta^{\prime}\right\}<1$. At the same time, as the function $f$ is assumed to be continuous according to the assumption $\mathbf{H}_{1}$, the operator $S_{1}$ is also continuous on $\mathscr{B}_{\varepsilon}$. To complete the proof of this theorem, it remains to show that $S_{1}$ is compact. In regard to Arzela-Ascoli theorem, it is enough to show that $S_{1} \mathscr{B}_{\varepsilon}$ is uniformly bounded and equicontinuous. For the boundedness, one can easily verify that $S_{1} z$ satisfies the following perspective.

$$
\left\|S_{1} z\right\|_{\mathscr{P}} \leq\|q\| \max \left\{\Delta(s+r), \Delta^{\prime}(s+r)\right\} .
$$

In the meantime, to show that $S_{1}$ is an equicontinuous operator, let $t_{1}, t_{2} \in[0, T]$ such that $t_{1}<t_{2}$. Then, we have

$$
\begin{aligned}
\left|S_{1} z\left(t_{2}\right)-S_{1} z\left(t_{1}\right)\right| \leq & \frac{1}{\Gamma(s+r)} \int_{0}^{t_{1}}\left|\left(t_{2}-\tau\right)^{s+r-1}-\left(t_{1}-\tau\right)^{s+r-1}\right| \\
& \times\left|f\left(\tau, z(\tau), D^{\rho} z(\tau)\right)\right| d \tau \\
& +\frac{1}{\Gamma(s+r)} \int_{t_{1}}^{t_{2}}\left(t_{2}-\tau\right)^{s+r-1}\left|f\left(\tau, z(\tau), D^{\rho} z(\tau)\right)\right| d \tau \\
& +\frac{\left|\chi_{1}\left(t_{1}\right)-\chi_{1}\left(t_{2}\right)\right|}{\Gamma(s+r)} \int_{0}^{T}(T-\tau)^{s+r-1}\left|f\left(\tau, z(\tau), D^{\rho} z(\tau)\right)\right| d \tau \\
& +\frac{\left|\chi_{2}\left(t_{1}\right)-\chi_{2}\left(t_{2}\right)\right|}{\Gamma(s+r)} \int_{0}^{\zeta}(\zeta-\tau)^{s+r-1}\left|f\left(\tau, z(\tau), D^{\rho} z(\tau)\right)\right| d \tau .
\end{aligned}
$$

Again, using condition $\mathbf{H}_{2}$ to turn the inequality above to be in the form

$$
\begin{aligned}
\left|S_{1} z\left(t_{2}\right)-S_{1} z\left(t_{1}\right)\right| \leq & \frac{\|q\|}{\Gamma(s+r)}\left[\int _ { 0 } ^ { t _ { 1 } } \left(\left(t_{2}-\tau\right)^{s+r-1}\right.\right. \\
& \left.-\left(t_{1}-\tau\right)^{s+r-1}\right) d \tau+\int_{t_{1}}^{t_{2}}\left(t_{2}-\tau\right)^{s+r-1} d \tau \\
& +\frac{\left|t_{2}^{s}\left(t_{2}-\zeta\right)-t_{1}^{s}\left(t_{1}-\zeta\right)\right|}{T^{s}(T-\zeta)} \int_{0}^{T}(T-\tau)^{s+r-1} d \tau \\
& \left.+\frac{\left|t_{2}^{s}\left(T-t_{2}\right)-t_{1}^{s}\left(T-t_{1}\right)\right|}{\zeta^{s}(T-\zeta)} \int_{0}^{\zeta}(\zeta-\tau)^{s+r-1} d \tau\right] .
\end{aligned}
$$

Consequently, the above inequality leads us to another one of the form

$$
\begin{aligned}
& \left|S_{1} z\left(t_{2}\right)-S_{1} z\left(t_{1}\right)\right| \\
& \quad \leq \frac{\|q\|}{\Gamma(s+r+1)} \\
& \quad \cdot\left[t_{2}^{s+r}-t_{1}^{s+r}+\frac{\left(t_{2}^{s+1}-t_{1}^{s+1}\right)\left(T^{r}+\zeta^{r}\right)+\zeta T\left(t_{2}-t_{1}\right)\left(T^{r-1}+\zeta^{r-1}\right)}{T-\zeta}\right] .
\end{aligned}
$$

Observe that as $t_{2} \rightarrow t_{1}$, the right-hand side goes to zero uniformly. This means that such side does not depend never on $z$. Therefore, as per Arzela-Ascoli theorem, the operator $S_{1}$ is equicontinuous. Hence, in view of Krasnoselskii theorem, we conclude that (1)-(2) has at least one solution.

In the next argument, we will investigate the uniqueness of solution for the problem under consideration. Of course, Banach fixed point theorem, reported in Theorem 7, will be utilized in this investigation. However, the following result is established for achieving that purpose.

Theorem 14. Suppose $\mathbf{H}_{1}$ and $\mathbf{H}_{3}$ hold. If

$$
\begin{aligned}
\Psi= & \left(M_{1}+M_{2}\right) \max \left\{\Delta(s+r), \Delta^{\prime}(s+r)\right\} \\
& +\max \left\{\Delta, \Delta^{\prime}\right\}<1 .
\end{aligned}
$$

Then, the problem given in (1)-(2) has a unique solution on $[0, T]$. 
Proof. Consider the set $\mathscr{B}_{\varepsilon}$ as given in (45) with

$$
\varepsilon \geq \frac{W \max \left\{\Delta(s+r), \Delta^{\prime}(s+r)\right\}}{1-\Psi},
$$

where $W=\sup _{0 t T}|f(t, 0,0)|$. For each $t \in[0, T]$ and $z \in \mathscr{B}_{\varepsilon}$, one might estimate the term $\left|f\left(t, z(t),{ }^{c} D^{\rho} z(t)\right)\right|$ as follows:

$$
\begin{aligned}
\left|f\left(t, z(t),{ }^{c} D^{\rho} z(t)\right)\right| & =\left|f\left(t, z(t),{ }^{c} D^{\rho} z(t)\right)-f(t, 0,0)+f(t, 0,0)\right| \\
& \leq\left|f\left(t, z(t),{ }^{c} D^{\rho} z(t)\right)-f(t, 0,0)\right|+|f(t, 0,0)| \\
& \leq M_{1}|z(t)|+M_{2}\left|{ }^{c} D^{\rho} z(t)\right|+W \\
& \leq\left(M_{1}+M_{2}\right)\|z\|_{\mathscr{P}}+W \leq\left(M_{1}+M_{2}\right) \varepsilon+\mathrm{W} .
\end{aligned}
$$

The next step is now to show that $S \mathscr{B}_{\varepsilon} \subseteq \mathscr{B}_{\varepsilon}, \forall z \in \mathscr{B}_{\varepsilon}$. Based on the arguments given in the previous theorem, one can obtain the following assertions.

$$
\begin{gathered}
\left|S_{1} z(t)\right| \leq\left(\left(M_{1}+M_{2}\right) \varepsilon+W\right) \Delta(s+r), \\
\left|{ }^{c} D^{\rho} S_{1} z(t)\right| \leq\left(\left(M_{1}+M_{2}\right) \varepsilon+W\right) \Delta^{\prime}(s+r), \\
\left|S_{2} z(t)\right| \leq \Delta \varepsilon, \\
\left|{ }^{c} D^{\rho} S_{2} z(t)\right| \leq \Delta^{\prime} \varepsilon .
\end{gathered}
$$

Applying the norm over $t$ gives

$$
\begin{aligned}
& \left\|S_{1} z\right\|_{\mathscr{P}} \leq\left(\left(M_{1}+M_{2}\right) \varepsilon+W\right) \max \left\{\Delta(s+r), \Delta^{\prime}(s+r)\right\} \\
& \left\|S_{2} z\right\|_{\mathscr{P}} \leq \varepsilon \max \left\{\Delta, \Delta^{\prime}\right\} .
\end{aligned}
$$

Therefore,

$$
\begin{aligned}
\|S z\|_{\mathscr{P}} \leq & \left\|S_{1} z\right\|_{\mathscr{P}}+\left\|S_{2} z\right\|_{\mathscr{P}} \\
\leq & \left(\left(M_{1}+M_{2}\right) \varepsilon+W\right) \max \\
& \cdot\left\{\Delta(s+r), \Delta^{\prime}(s+r)\right\}+\varepsilon \max \left\{\Delta, \Delta^{\prime}\right\} \\
= & \Psi \varepsilon+W \max \left\{\Delta(s+r), \Delta^{\prime}(s+r)\right\} \leq \varepsilon .
\end{aligned}
$$

Now, in accordance to Theorem 7, we need to show that the mapping $S$ is contraction. In order to achieve this goal, we should notice that the operator $S_{2}$ is contraction as shown in the previous theorem. Hence, it remains to verify that $S_{1}$ is also contraction. For this purpose, let $z_{1}, z_{2} \in \mathscr{B}_{\mathcal{E}}, t \in[0, T]$ and consider $\left|S_{1} z_{1}(t)-S_{1} z_{2}(t)\right|$ as follows:

$$
\begin{aligned}
\left|S_{1} z_{1}(t)-S_{1} z_{2}(t)\right| \leq & I^{s+r} \mid f\left(t, z_{1}(t),{ }^{c} D^{\rho} z_{1}(t)\right) \\
& -f\left(t, z_{2}(t),{ }^{c} D^{\rho} z_{2}(t)\right) \mid \\
& +\left|\chi_{1}(t)\right| I^{s+r} \mid f\left(T, z_{1}(T),{ }^{c} D^{\rho} z_{1}(T)\right) \\
& -\mathrm{f}\left(T, z_{2}(T),{ }^{c} D^{\rho} z_{2}(T)\right) \mid \\
& +\left|\chi_{2}(t)\right| I^{s+r}|| f\left(\zeta, z_{1}(\zeta),{ }^{c} D^{\rho} z_{1}(\zeta)\right) \\
& -f\left(\zeta, z_{2}(\zeta),{ }^{c} D^{\rho} z_{2}(\zeta)\right) \mid .
\end{aligned}
$$

Using condition $\mathbf{H}_{3}$ yields

$$
\begin{aligned}
\left|S_{1} z_{1}(t)-S_{1} z_{2}(t)\right| \leq & \frac{1}{\Gamma(s+r)} \int_{0}^{t}(t-\tau)^{s+r-1} \\
& \cdot\left(M_{1}\left|z_{1}-z_{2}\right|+\left.M_{2}\right|^{c} D^{\rho} z_{1}-{ }^{c} D^{\rho} z_{2} \mid\right) d \tau \\
& +\frac{\left|\chi_{1}(t)\right|}{\Gamma(s+r)} \int_{0}^{T}(T-\tau)^{s+r-1} \\
& \cdot\left(M_{1}\left|z_{1}-z_{2}\right|+\left.M_{2}\right|^{c} D^{\rho} z_{1}-{ }^{c} D^{\rho} z_{2} \mid\right) d \tau \\
& +\frac{\left|\chi_{2}(t)\right|}{\Gamma(s+r)} \int_{0}^{\zeta}(\zeta-\tau)^{s+r-1} \\
& \cdot\left(M_{1}\left|z_{1}-z_{2}\right|+\left.M_{2}\right|^{c} D^{\rho} z_{1}-{ }^{c} D^{\rho} z_{2} \mid\right) d \tau, \\
\leq & \left(M_{1}\left|z_{1}(t)-z_{2}(t)\right|+\left.M_{2}\right|^{c} D^{\rho} z_{1}(t)\right. \\
& \left.-{ }^{c} D^{\rho} z_{2}(t) \mid\right) \Delta(s+r) .
\end{aligned}
$$

Similarly, we can obtain the following inequality:

$$
\begin{aligned}
\left|{ }^{c} D^{\rho} S_{1} z_{1}(t)-{ }^{c} D^{\rho} S_{1} z_{2}(t)\right| & \\
\leq & \left(M_{1}\left|z_{1}(t)-z_{2}(t)\right|+M_{2} \mid{ }^{c} D^{\rho} z_{1}(t)\right. \\
& \left.-{ }^{c} D^{\rho} z_{2}(t) \mid\right) \Delta^{\prime}(s+r) .
\end{aligned}
$$

Taking under the norm $\|\cdot\|_{\mathscr{P}}$ implies

$\left\|S_{1} z_{1}-S_{1} z_{2}\right\|_{\mathscr{P}} \leq\left(M_{1}+M_{2}\right) \max \left\{\Delta(s+r), \Delta^{\prime}(s+r)\right\}\left\|z_{1}-z_{2}\right\|_{\mathscr{P}}$.

Using the two deduced assertions in (54) and (68) implies

$$
\begin{aligned}
\left\|S z_{1}-S z_{2}\right\|_{\mathscr{P}} \leq & \left\|S_{1} z_{1}-S_{1} z_{2}\right\|_{\mathscr{P}}+\left\|S_{2} z_{1}-S_{2} z_{2}\right\|_{\mathscr{P}} \\
\leq & {\left[\left(M_{1}+M_{2}\right) \max \left\{\Delta(s+r), \Delta^{\prime}(s+r)\right\}\right.} \\
& \left.+\max \left\{\Delta, \Delta^{\prime}\right\}\right]\left\|z_{1}-z_{2}\right\|_{\mathscr{P}} \leq \Psi\left\|z_{1}-z_{2}\right\|_{\mathscr{P}}
\end{aligned}
$$

which shows that $S$ is a contraction mapping, and this finishes the proof.

In the same context, we introduce the following further result which also explores the existence matter of our main problem by taking into account, this time, Theorem 9 . 
Theorem 15. Suppose $\mathbf{H}_{1}$ and $\mathbf{H}_{4}$ hold. If

$\frac{\lambda}{\max \left\{\left(\|h\| \Psi\left(\|z\|_{\mathscr{P}}\right) \Delta(s+r)+\Delta\right),\left(\|h\| \Psi\left(\|z\|_{\mathscr{P}}\right) \Delta^{\prime}(s+r)+\Delta^{\prime}\right)\right\}}>1$

Then, the problem given in (1)-(2) has at least one solution on $[0, T]$, for some $\lambda, \varepsilon>0$, where $\Delta, \Delta, \Delta(\cdot)$ and $\Delta^{\prime}(\cdot)$ are defined, respectively, in (36), (37), (38) and (39).

Proof. Suppose $\mathcal{O}=\left\{z \in \mathscr{C}:{ }^{c} D^{\rho} z \in \mathscr{C},\|z\|_{\mathscr{P}}<\lambda\right\}$ is an open subset of the Banach space in $\mathscr{C}=C\left([0, T], \mathbb{R}^{+}\right)$. Assume that $S: \bar{O} \rightarrow \in \mathscr{C}$ is an operator defined as given in (26) with its fractional derivatives in (27), which are also previously defined as a sum of two operators $S_{1}$ and $S_{2}$. Observe that $S$ is continuous since $S_{1}$ and $S_{2}$ are so. From this point, we find that, it is the right time, to apply on Theorem 9. For that purpose and according to Arzela-Ascoli theorem, we need to show that $S$ is compact. In other words, we need to show that such operator is bounded and also equicontinuous. For the boundedness, let $t \in[0, T]$ and consider the term $|S z(t)|$ as follows:

$$
\begin{aligned}
|S z(t)| \leq & I^{s+r}\left|f\left(t, z(t), D^{\rho} z(t)\right)\right|+|\ell| I^{s}|z(t)| \\
& +\left|\chi_{1}(t)\right|\left[|\alpha| I|z(T)|+I^{s+r}\left|f\left(T, z(T), D^{\rho} z(T)\right)\right|\right. \\
& \left.+|\ell| I^{s}|z(T)|\right]+\left|\chi_{2}(t)\right|[|\beta| I|z(\zeta)| \\
& \left.+I^{s+r}\left|f\left(\zeta, z(\zeta), D^{\rho} z(\zeta)\right)\right|+|\ell| I^{s}|z(\zeta)|\right] .
\end{aligned}
$$

Using condition $\mathbf{H}_{4}$ yields

$$
\begin{aligned}
|S z(t)| \leq & \frac{\|h\| \Psi\left(\|z\|_{\mathscr{P}}\right)}{\Gamma(s+r)} \int_{0}^{t}(t-\tau)^{s+r-1} d \tau \\
& +\frac{|\ell|\|z\|}{\Gamma(s+1)} t^{s}+\left|\chi_{1}(t)\right|\left[|\alpha| T\|z\|_{\mathscr{P}}\right. \\
& +\frac{\|h\| \Psi\left(\|z\|_{\mathscr{P}}\right)}{\Gamma(s+r)} \int_{0}^{T}(T-\tau)^{s+r-1} d \tau \\
& \left.+\frac{|\ell|\|z\|}{\Gamma(s+1)} T^{s}\right]+\left|\chi_{2}(t)\right|\left[|\beta| \zeta\|\|_{\mathscr{P}}\right. \\
& \left.+\frac{\|h\| \Psi\left(\|z\|_{\mathscr{P}}\right)}{\Gamma(s+r)} \int_{0}^{\zeta}(\zeta-\tau)^{s+r-1} d \tau+\frac{|\ell|\|z\|}{\Gamma(s+1)} \zeta^{s}\right] .
\end{aligned}
$$
assertion.

Consequently, the above inequality leads to the following

$$
\|S z(t)\|=\sup _{0 t T}|S z(t)| \leq\|h\| \Psi\left(\|z\|_{\mathscr{P}}\right) \Delta(s+r)+\|z\|_{\mathscr{P}} \Delta .
$$

Similarly, we can easily obtain the following other assertion.

$$
\left\|{ }^{c} D^{\rho} S z\right\|=\sup _{0 t T}\left|{ }^{c} D^{\rho} S z(t)\right| \leq\|h\| \Psi\left(\|z\|_{\mathscr{P}}\right) \Delta^{\prime}(s+r)+\|z\|_{\mathscr{P}} \Delta^{\prime} .
$$

By the last two inequalities, one can deduce that the operator $S$ is bounded. On the other hand, to prove that this operator is also equicontinuous, we should recall that it has been previously formulated as a sum of two operators $S_{1}$ and $S_{2}$, with noting that the first one, $S_{1}$, has appeared as an equicontinuous operator through the proof of Theorem 13.. Therefore, to complete the proof of this theorem, it remains to show that $S_{2}$ is also equicontinuous. In order to achieve this objective, let $t_{1}, t_{2} \in[$ $0, T]$ such that $t_{1}<t_{2}$. Then, we have

$$
\begin{aligned}
\left|S_{2} z\left(t_{2}\right)-S_{2} z\left(t_{1}\right)\right| \leq & \frac{|\ell|}{\Gamma(s)} \times\left[\int _ { 0 } ^ { t _ { 1 } } \left(\left(t_{1}-\tau\right)^{s-1}\right.\right. \\
& \left.-\left(t_{2}-\tau\right)^{s-1}\right)|z(\tau)| d \tau \\
& \left.+\int_{t_{1}}^{t_{2}}\left(t_{2}-\tau\right)^{s-1}|z(\tau)| d \tau\right] \\
& +\left|\chi_{1}\left(t_{2}\right)-\chi_{1}\left(t_{1}\right)\right|\left[|\alpha| \int_{0}^{T}|z(\tau)| d \tau\right. \\
& \left.+\frac{|\ell|}{\Gamma(s)} \int_{0}^{T}(T-\tau)^{s-1}|z(\tau)| d \tau\right] \\
& +\left|\chi_{2}\left(t_{2}\right)-\chi_{2}\left(t_{1}\right)\right|\left[|\beta| \int_{0}^{\zeta}|z(\tau)| d \tau\right. \\
& \left.+\frac{|\ell|}{\Gamma(s)} \int_{0}^{\zeta}(\zeta-\tau)^{s-1}|z(\tau)| d \tau\right] .
\end{aligned}
$$

Actually, the above inequality leads to the following other one

$$
\begin{aligned}
\left|S_{2} z\left(t_{2}\right)-S_{2} z\left(t_{1}\right)\right| \leq & \| z \mid\left[\frac { | \ell | } { \Gamma ( s + 1 ) } \left(2\left(t_{2}-t_{1}\right)^{s}\right.\right. \\
& \left.+t_{1}^{s}-t_{2}^{s}\right)+\frac{\left|t_{2}^{s}\left(t_{2}-\zeta\right)-t_{1}^{s}\left(t_{1}-\zeta\right)\right|}{T^{s}(T-\zeta)} \\
& \cdot\left(|\alpha| T+\frac{|\ell| T^{s}}{\Gamma(s+1)}\right) \\
& +\frac{\left|t_{2}^{s}\left(T-t_{2}\right)-t_{1}^{s}\left(T-t_{1}\right)\right|}{\zeta^{s}(T-\zeta)} \\
& \left.\cdot\left(|\beta| \zeta+\frac{|\ell| \zeta^{s}}{\Gamma(s+1)}\right)\right] \\
\leq & \|z\|\left[\frac{2|\ell|}{\Gamma(s+1)}\left(t_{2}-t_{1}\right)^{s}\right. \\
& +\frac{t_{2}^{s+1}-t_{1}^{s+1}+\zeta\left(t_{2}^{s}-t_{1}^{s}\right)}{T^{s}(T-\zeta)} \\
& \cdot\left(|\alpha| T+\frac{|\ell| T^{s}}{\Gamma(s+1)}\right) \\
& +\frac{t_{2}^{s+1}-t_{1}^{s+1}+T\left(t_{2}^{s}-t_{1}^{s}\right)}{\zeta^{s}(T-\zeta)} \\
& \left.\cdot\left(|\beta| \zeta+\frac{|\ell| \zeta^{s}}{\Gamma(s+1)}\right)\right] .
\end{aligned}
$$


One observes that the right-hand side of inequality above goes to zero uniformly as $t_{2} \rightarrow t_{1}$. This, however, implies that $S_{2}$ is definitely equicontinuous via Arzela-Ascoli theorem. The last step in this proof that should be considered is showing the boundedness of the solution of the equation $z=\gamma S z$, where $\gamma \in[0,1]$. In this regard, let $t \in[0, T]$ and $z$ be such solution, then by considering $(4.12,4.12)$, we have

$$
\begin{aligned}
\|z\|_{\mathscr{P}}= & \gamma\|S z\|_{\mathscr{P}} \\
\leq & \|S z\|_{\mathscr{P}} \\
= & \max \left\{\|S z\|,\left\|^{c} D^{\rho} S z\right\|\right\} \\
\leq & \|h\| \Psi\left(\|z\|_{\mathscr{P}}\right) \max \\
& \cdot\left\{\Delta(s+r), \Delta^{\prime}(s+r)\right\}+\|z\|_{\mathscr{P}} \max \left\{\Delta, \Delta^{\prime}\right\} .
\end{aligned}
$$

Using the previous inequality allows one to reveal the following inequality.

$\frac{\|z(t)\|_{\mathscr{P}}}{\|h\| \Psi\left(\|z\|_{\mathscr{P}}\right) \max \left\{\Delta(s+r), \Delta^{\prime}(s+r)\right\}+\|z\|_{\mathscr{P}} \max \left\{\Delta, \Delta^{\prime}\right\}} \leq 1$.

Obviously, due to $\|z(t)\|_{\mathscr{P}}<\lambda$ as previously defined in $\mathcal{O}$, then the last assertion contradicts assumption (70). Hence, by Theorem 9, one can conclude that $S$ has a fixed point $z \in \overline{\mathcal{O}}$, which finishes the proof.

\section{Illustrative Examples}

This section introduces three examples to demonstrate the proposed results related to the main issue in this work. For simplicity in calculations, these three examples are unified under the same values of our problem's parameters (1)-(2). In other words, we take $r=8 / 5, s=2 / 3, \rho=1 / 2, \zeta=1 / 2, T$ $=1, \ell=1 / 75, \alpha=1 / 150$ and $\beta=3 / 200$. Setting these values in the terms (19), (20), (30) and (31) simplifies their expressions to be in the following forms $\chi_{1}=1, \chi_{2}=1.03413, \chi_{3}$ $=1.80715$ and $\chi_{4}=1.80456$. Accordingly, the following terms are also estimated via (36)-(39) as $\Delta(s=2 / 3)=2.937$, $\Delta(s+r=34 / 15)=0.854, \Delta=0.054, \Delta^{\prime}(s=2 / 3)=4.339, \Delta^{\prime}($ $s+r=34 / 15)=1.455$ and $\Delta^{\prime}=0.083$.

Example 1. Consider the following problem.

$D^{8 / 5}\left(D^{2 / 3}+\frac{1}{75}\right) z(t)=\frac{e^{-t}}{\left(7+e^{t}\right)}\left(\frac{\left|z(t) D^{1 / 2} z(t)\right|}{1+\left|z(t) D^{1 / 2} z(t)\right|}\right), t \in[0,1]$,

subject to the following conditions

$z(0)=0, \quad z(1)=\frac{1}{150} \int_{0}^{1} z(u) d u, \quad z\left(\frac{1}{2}\right)=\frac{3}{200} \int_{0}^{1 / 2} z(u) d u$.
Comparing (79)-(80) with (1)-(2) yields the following continuous function on $[0,1]$

$$
f\left(t, z(t), D^{1 / 2} z(t)\right)=\frac{e^{-t}}{\left(7+e^{t}\right)}\left(\frac{\left|z(t) D^{1 / 2} z(t)\right|}{1+\left|z(t) D^{1 / 2} z(t)\right|}\right) .
$$

Note that $\mathbf{H}_{1}$ is satisfied, and, on the other hand, we have

$$
\left|f\left(t, z(t), D^{1 / 2} z(t)\right)\right| \leq\left|\frac{e^{-t}}{\left(7+e^{t}\right)}\right|\left|\frac{\left|z(t) D^{1 / 2} z(t)\right|}{1+\left|z(t) D^{1 / 2} z(t)\right|}\right|,
$$

which implies

$$
\left|f\left(t, z(t), D^{1 / 2} z(t)\right)\right| \leq q(t)=\frac{e^{-t}}{7+e^{t}} .
$$

Therefore, condition $\mathbf{H}_{2}$ is also satisfied with $\|q\|=1 / 8$. Now, based on the above estimations, one can verify the following statement:

$$
\max \left\{\Delta, \Delta^{\prime}\right\}=0.083<1 .
$$

This means that the problem given in (79)-(80) has a unique solution on $[0,1]$, as per Theorem 13..

Example 2. Consider the following problem:

$$
\begin{aligned}
D^{8 / 5}\left(D^{2 / 3}+\frac{1}{75}\right) z(t) \\
=\frac{\ln t}{2 t} \cdot \frac{|z(t)|}{2+|z(t)|} \\
\quad+\frac{\left|D^{1 / 2} z(t)\right|}{10(1+t)^{2}\left(\left|D^{1 / 2} z(t)\right|+1\right)},
\end{aligned}
$$

subject to the same conditions given in (80). One can notice that the function $f$ has the form

$f\left(t, z(t), D^{1 / 2} z(t)\right)=\frac{\ln t}{2 t} \cdot \frac{|z(t)|}{2+|z(t)|}+\frac{\left|D^{1 / 2} z(t)\right|}{10(1+t)^{2}\left(\left|D^{1 / 2} z(t)\right|+1\right)}$,

which satisfies $\mathbf{H}_{1}$ and implies

$$
\begin{aligned}
& \left|f\left(t, z_{1}(t), D^{1 / 2} z_{1}(t)\right)-f\left(t, z_{2}(t), D^{1 / 2} z_{2}(t)\right)\right| \\
& \quad \leq \frac{1}{4}\left|z_{1}-z_{2}\right|+\frac{1}{10}\left|D^{1 / 2} z_{1}-D^{1 / 2} z_{2}\right| .
\end{aligned}
$$

This definitely means that condition $\mathbf{H}_{3}$ is hold, with $M_{1}=1 / 4$ and $M_{2}=1 / 10$. Besides, one can have the following assertion.

$$
\begin{aligned}
\Psi= & \left(M_{1}+M_{2}\right) \max \left\{\Delta(s+r), \Delta^{\prime}(s+r)\right\} \\
& +\max \left\{\Delta, \Delta^{\prime}\right\}=0.5926<1,
\end{aligned}
$$


which implies that the problem (85) has a unique solution on $[0,1]$ according to Theorem 14 .

Example 3. Consider the following problem.

$$
\begin{aligned}
& D^{8 / 5}\left(D^{2 / 3}+\frac{1}{75}\right) z(t) \\
& =\frac{e^{-t^{4}} \max \left\{\sup _{0 \leq t \leq 1}|z(t)|,\left.\sup _{0 \leq t \leq 1}\right|^{c} D^{1 / 2}(z(t)) \mid\right\}}{5 \sqrt{1+t^{2}}},
\end{aligned}
$$

subject to the same conditions given in (80). In this example, we have the function $f$ is of the form

$$
\begin{aligned}
f(t, & \left.z(t), D^{1 / 2} z(t)\right) \\
& =\frac{e^{-t^{4}} \max \left\{\sup _{0 \leq t \leq 1}|z(t)|,\left.\sup _{0 \leq t \leq 1}\right|^{c} D^{1 / 2}(z(t)) \mid\right\}}{5 \sqrt{1+t^{2}}} .
\end{aligned}
$$

In fact, the above function satisfies $\mathbf{H}_{1}$ and implies

$$
\left|f\left(t, z(t), D^{1 / 2} z(t)\right)\right| \leq h(t) \Psi\left(\|z\|_{\mathscr{P}}\right)
$$

where $h(t)=e^{-t^{4}} / 5 \sqrt{1+t^{2}}$ and $\Psi\left(\|z\|_{\mathscr{P}}\right)=\|z\|_{\mathscr{P}}<\lambda$, in which $\lambda$ is assumed as given in (70). In other words, condition $\mathbf{H}_{4}$ is satisfied with $\|h\|=1 / 5$, while the assumption given in (70) will be hold if

$$
\max \left\{\left(0.1708\|z\|_{\mathscr{P}}+0.054\right),\left(0.291\|z\|_{\mathscr{P}}+0.083\right)\right\}<\lambda .
$$

This, consequently, implies $\lambda>0.117$. It means that the problem (89), in view of Theorem 15., has at least one solution on $[0,1]$, for $\lambda>0.117$.

\section{Conclusion}

The existence and uniqueness of solution for the fractionalorder Langevin equation have been studied and explored in this article. This problem has been addressed subject to novel three-point boundary conditions involving two nonlocal integrals. In view of this study, some new applicable conditions and results have been established associated with our main target. These results have been illustrated through three numerical examples.

\section{Data Availability}

There is no data available.

\section{Conflicts of Interest}

The authors declare that they have no conflicts of interest.

\section{Acknowledgments}

This project was funded by the Deanship of Scientific Research (DSR), King Abdulaziz University, Jeddah, under grant No. D-054-130-1438. The authors, therefore, acknowledge with thanks to the DSR for the technical and financial support.

\section{References}

[1] A. Salem and M. Alnegga, "Fractional Langevin equations with multi-point and non-local integral boundary conditions," Cogent Mathematics \& Statistics, vol. 7, no. 1, 2020.

[2] A. Salem and A. Al-Dosari, "Existence results of solution for fractional Sturm-Liouville inclusion involving composition with multi-maps," Journal of Taibah University for Science, vol. 14, no. 1, pp. 721-733, 2020.

[3] H. Sun, W. Chen, and Y. Chen, "Variable-order fractional differential operators in anomalous diffusion modeling," Physica A: Statistical Mechanics and its Applications, vol. 388, no. 21, pp. 4586-4592, 2009.

[4] Y. Wang, L. Gu, Y. Xu, and X. Cao, "Practical tracking control of robot manipulators with continuous fractional-order nonsingular terminal sliding mode," IEEE Transactions on Industrial Electronics, vol. 63, no. 10, pp. 6194-6204, 2016.

[5] J. Machado and M. E. Mata, "A fractional perspective to the bond graph modelling of world economies," Nonlinear Dynamics, vol. 80, no. 4, pp. 1839-1852, 2015.

[6] M. R. B. Shahriyar, F. Ismail, S. Aghabeigi, A. Ahmadian, and S. Salahshour, "An eigenvalue-eigenvector method for solving a system of fractional differential equations with uncertainty," Mathematical Problems in Engineering, vol. 2013, Article ID 579761, 11 pages, 2013.

[7] S. Salahshour, A. Ahmadian, and C. S. Chan, "Successive approximation method for Caputo q-fractional IVPs," Communications in Nonlinear Science and Numerical Simulation, vol. 24, no. 1-3, pp. 153-158, 2015.

[8] A. Ahmadian, C. S. Chan, S. Salahshour, and V. Vaitheeswaran, "FTFBE: a numerical approximation for fuzzy time-fractional Bloch equation," in 2014 IEEE International Conference on Fuzzy Systems (FUZZ-IEEE), pp. 418423, Beijing, China, 2014.

[9] A. Ahmadian, S. Salahshour, M. Ali-Akbari, F. Ismail, and D. Baleanu, "A novel approach to approximate fractional derivative with uncertain conditions," Chaos, Solitons \& Fractals, vol. 104, pp. 68-76, 2017.

[10] A. Salem, F. Alzahrani, and A. Al-Dosari, "Attainability to solve fractional differential inclusion on the half line at resonance," Complexity, vol. 2020, Article ID 9609108, 13 pages, 2020.

[11] H. Baghani, "An analytical improvement of a study of nonlinear Langevin equation involving two fractional orders in different intervals," Journal of Fixed Point Theory and Applications, vol. 21, no. 4, pp. 1-11, 2019.

[12] Z. Bai and W. Sun, "Existence and multiplicity of positive solutions for singular fractional boundary value problems," Computers \& Mathematcs with Applications, vol. 63, no. 9, pp. 1369-1381, 2012.

[13] S. Liang and J. Zhang, "Existence of multiple positive solutions for $m$-point fractional boundary value problems on an infinite 
interval," Mathematical and Computer Modelling, vol. 54, no. 5-6, pp. 1334-1346, 2011.

[14] A. Salem, F. Alzahrani, and L. Almaghamsi, "Fractional Langevin equations with nonlocal integral boundary conditions," Mathematics, vol. 7, no. 5, 2019.

[15] A. Salem and M. Alnegga, "Measure of noncompactness for hybrid Langevin fractional differential equations," Axioms, vol. 9, no. 2, 2020.

[16] W. T. Coffey, Y. P. Kalmykov, and J. T. Waldron, The Langevin Equation: With Applications to Stochastic Problems in Physics, Chemistry and Electrical Engineering, vol. 14, WORLD SCIENTIFIC, Singapore, 2004.

[17] B. J. West and S. Picozzi, "Fractional Langevin model of memory in financial time series," Physical Review E, vol. 65, no. 3, 2002.

[18] P. C. Hohenberg and B. I. Halperin, "Theory of dynamic critical phenomena," Reviews of Modern Physics, vol. 49, no. 3, pp. 435-479, 1977.

[19] F. Mainradi and P. Pironi, "The fractional Langevin equation: Brownian motion revisted," Extracta Mathematicae, vol. 10, pp. 140-154, 1996.

[20] C. Zhai and P. Li, "Nonnegative solutions of initial value problems for Langevin equations involving two fractional orders," Mediterranean Journal of Mathematics, vol. 15, no. 4, 2018.

[21] H. Baghani, "Existence and uniqueness of solutions to fractional Langevin equations involving two fractional orders," Journal of Fixed Point Theory and Applications, vol. 20, no. 2, 2018.

[22] H. Fazli and J. J. Nieto, "Fractional Langevin equation with anti-periodic boundary conditions," Chaos, Solitons and Fractals, vol. 114, pp. 332-337, 2018.

[23] B. Datsko and V. Gafiychuk, "Complex nonlinear dynamics in subdiffusive activator-inhibitor systems," Communications in Nonlinear Science and Numerical Simulation, vol. 17, no. 4, pp. 1673-1680, 2012.

[24] B. Datsko and V. Gafiychuk, "Complex spatio-temporal solutions in fractional reaction-diffusion systems near a bifurcation point," Fractional Calculus and Applied Analysis, vol. 21, no. 1, pp. 237-253, 2018.

[25] G. Wang and M. Tokuyama, "Nonequilibrium statistical description of anomalous diffusion," Physica A: Statistical Mechanics and its Applications, vol. 265, no. 3-4, pp. 341351, 1999.

[26] E. Lutz, "Fractional Langevin equation," Physical Review E, vol. 64 , no. $5,2001$.

[27] W. Yukunthorn, S. K. Ntouyas, and J. Tariboon, "Nonlinear fractional Caputo Langevin equation with nonlocal Riemann Liouville fractional integral conditions," Advances in Difference Equations, vol. 2014, no. 1, Article ID 315, 2014.

[28] B. Ahmad, M. Alghanmi, A. Alsaedi, H. M. Srivastava, and S. N. Ntouyas, "The Langevin equation in terms of generalized Liouville-Caputo derivatives with nonlocal boundary conditions involving a generalized fractional integral," Mathematics, vol. 7, no. 6, 2019.

[29] A. Salem, F. Alzahrani, and M. Alnegga, "Coupled system of non-linear fractional Langevin equations with multi-point and nonlocal integral boundary conditions," Mathematical Problems in Engineering, vol. 2020, Article ID 7345658, 15 pages, 2020.

[30] A. Salem, F. Alzahrani, and B. Alghamdi, "Langevin equation involving two fractional orders with three-point boundary conditions," Differential and Integral Equations, vol. 33, pp. 163-180, 2020.

[31] N. I. Mahmudov, "Fractional Langevin type delay equations with two fractional derivatives," Applied Mathematics Letters, vol. 103, article 106215, 2020.

[32] H. Baghani and J. J. Nieto, "On fractional Langevin equation involving two fractional orders in different intervals," Nonlinear Analysis: Modelling and Control, vol. 24, no. 6, pp. 884897, 2019.

[33] A. Salem and B. Alghamdi, "Multi-point and anti-periodic conditions for generalized Langevin equation with two fractional orders," Fractal and Fractional, vol. 3, no. 4, 2019.

[34] O. Baghani, "On fractional Langevin equation involving two fractional orders," Communications in Nonlinear Science and Numerical Simulation, vol. 42, pp. 675-681, 2017.

[35] M. Benchohra and J. Lazreg, "Existence and uniqueness results for nonlinear implicit fractional differential equations with boundary conditions," Romanian Journal of Mathematics and Computer Science, vol. 4, no. 1, pp. 60-72, 2014.

[36] G. M. N’Guérékata, “A Cauchy problem for some fractional abstract differential equation with non local conditions," Nonlinear Analysis: Theory, Methods \& Applications, vol. 70, no. 5, pp. 1873-1876, 2009.

[37] A. A. Kilbas, H. M. Srivastava, and J. J. Trujillo, “Theory and Applications of Fractional Differential Equations," in NorthHolland Mathematics Studies, vol. 204, Elsevier Science B. V, Amsterdam, 2006.

[38] T. A. Burton, "A fixed-point theorem of Krasnoselskii," Applied Mathematics Letters, vol. 11, no. 1, pp. 85-88, 1998.

[39] A. Granas and J. Dugundji, Fixed Point Theory, Springer, New York, 2003.

[40] S. Tate and H. T. Dinde, "Boundary value problems for nonlinear implicit fractional differential equations," Journal Nonlinear Analysis and Application, vol. 2019, no. 2, pp. 29-40, 2019. 